\title{
Dietary Omega-3 Fatty Acids Modulate Large-Scale Systems Organization in the Rhesus Macaque Brain
}

\author{
David S. Grayson, ${ }^{1,2}$ Christopher D. Kroenke, ${ }^{2,3,6}$ Martha Neuringer, ${ }^{4,6}$ and Damien A. Fair ${ }^{2,3,5}$ \\ ${ }^{1}$ Center for Neuroscience, University of California, Davis, California 95616, ${ }^{2}$ Department of Behavioral Neuroscience, ${ }^{3}$ Advanced Imaging Research Center, \\ ${ }^{4}$ Casey Eye Institute, and ${ }^{5}$ Department of Psychiatry, Oregon Health and Science University, Portland, Oregon 97239, and ${ }^{6}$ Division of Neuroscience, Oregon \\ National Primate Research Center, Beaverton, Oregon 97006
}

Omega-3 fatty acids are essential for healthy brain and retinal development and have been implicated in a variety of neurodevelopmental disorders. This study used resting-state functional connectivity MRI to define the large-scale organization of the rhesus macaque brain and changes associated with differences in lifetime $\omega$ - 3 fatty acid intake. Monkeys fed docosahexaenoic acid, the long-chain $\omega$-3 fatty acid abundant in neural membranes, had cortical modular organization resembling the healthy human brain. In contrast, those with low levels of dietary $\omega-3$ fatty acids had decreased functional connectivity within the early visual pathway and throughout higher-order associational cortex and showed impairment of distributed cortical networks. Our findings illustrate the similarity in modular cortical organization between the healthy human and macaque brain and support the notion that $\omega$ - 3 fatty acids play a crucial role in developing and/or maintaining distributed, large-scale brain systems, including those essential for normal cognitive function.

Key words: brain organization; DHA; functional connectivity; macaque development; omega-3 fatty acids; visual pathway

\section{Introduction}

Omega-3 fatty acids ( $\omega$-3FAs) are essential for healthy brain development, but over the last 50 years, the content of $\omega$-3FAs in the Western diet has declined (Simopoulos, 2011). Docosahexaenoic acid (DHA), a long-chain $\omega$-3FA, is the most abundant polyunsaturated fatty acid in membranes of the mammalian retina and cerebral gray matter (Fliesler and Anderson, 1983; Innis, 2007). Importantly, it must be acquired through the diet or from endogenous conversion from $\alpha$-linolenic acid (ALA). DHA plays a major role in visual development, promoting the survival of retinal photoreceptors and pigment epithelial cells (Bazan, 2006) and stabilizing retinal projections to the lateral geniculate nucleus (LGN) and superior colliculus (SC; de Velasco et al., 2012). The importance of dietary $\omega$-3FAs for the development of visual acuity has been demonstrated in both humans and nonhuman primates (Neuringer et al., 1986; SanGiovanni et al., 2000; O'Connor et al., 2001), a finding that led to the addition of DHA to infant formulas worldwide.

\footnotetext{
Received July 17, 2013; revised Nov. 21, 2013; accepted Dec. 18, 2013

Author contributions: D.S.G., C.D.K., M.N., and D.A.F. designed research; D.S.G. and C.D.K. performed research; D.S.G. and D.A.F. contributed unpublished reagents/analytic tools; D.S.G. analyzed data; D.S.G., M.N., and D.A.F. wrote the paper.

This work was supported by the Oregon Clinical and Translational Research Institute and NIH (Grants UL1 RR024140 and P510D011092 to D.A.F., C.D.K., M.N.; Grant K99/R00 MH091238 to D.A.F.; and Grants EY13199 and DK29930 to M.N.); and the Foundation Fighting Blindness (to M.N.). We thank Laurie Renner, Alison Weiss, and Vincent Warren for their help with animal sedation and MRI scanning and Daniel Sussman for helpful discussions regarding data analyses.

The authors declare no competing financial interests.

Correspondence should be addressed to David Grayson, Center for Neuroscience, University of California, 1544 Newman Court, Davis, CA 95616. E-mail: dgrayson@ucdavis.edu.

DOI:10.1523/JNEUROSCI.3038-13.2014

Copyright $\odot 2014$ the authors $\quad 0270-6474 / 14 / 342065-10 \$ 15.00 / 0$
}

Growing evidence suggests that $\omega$-3FAs regulate brain development and function beyond the visual system. Human and animal models suggest that dietary deficiency of $\omega$-3FAs impairs learning and memory (Moriguchi et al., 2000; Wainwright, 2002), whereas DHA supplementation positively affects various forms of cognition across development (Hoffman et al., 2009; Ryan et al., 2010; Luchtman and Song, 2013). These findings suggest that DHA may modulate the coordination of large-scale brain systems involved in cognitive function and/or higher-order processing. However, characterizing specific and causal effects of dietary DHA on distributed cortical connectivity has remained a major challenge.

We used noninvasive resting-state functional connectivity MRI (rs-fcMRI) to investigate differences in global brain communication arising from lifelong $\omega$-3FA deficiency or DHA supplementation in the macaque. In humans, rs-fcMRI has been used to map distributed functional brain networks with distinct roles in sensory processing and in top-down cognitive control (Fair et al., 2007; Power et al., 2011; Yeo et al., 2011). Other work has validated the use of this measure in the macaque and has highlighted its potential to bridge information between human and animal models (Vincent et al., 2007; Margulies et al., 2009; Mantini et al., 2011). We previously characterized differences between rhesus macaques raised on an $\omega$-3FA-deficient diet and those raised on a high-ALA diet or a diet providing DHA and other long-chain polyunsaturated fatty acids. Deficient animals were shown to have delayed visual acuity development, altered rod photoreceptor function, and an $80-85 \%$ reduction in retinal and cortical DHA levels (Neuringer et al., 1986; Jeffrey et al., 2002; Jeffrey and Neuringer, 2009). Here, we assessed differences in visual pathway functional connectivity and large-scale cortical 
brain organization in a group of these animals, now 17-19 years of age. We first tested the hypothesis that deficits in connectivity within the early visual system would be observed in $\omega$-3FAdeficient animals. We then extended our analysis to more broadly characterize changes in functional connectivity throughout the cerebral cortex.

\section{Materials and Methods}

Subjects and experimental diets

Fifteen rhesus monkeys (Macaca mulatta) were fed one of three semisynthetic diets from birth until 17-19 years of age, as described previously (Jeffrey and Neuringer, 2009). In addition, during pregnancy, the mothers of the test animals had been maintained on the same diets as their offspring. During infancy, the diets were provided in liquid form, followed by solid diets thereafter. The three diets, which were otherwise identical, had different fat sources providing widely different levels of $\omega$-3Fas.

The $\omega$-3FA-deficient diet ( $n=7,4$ male, 3 female), with dietary fat supplied by a 1:1 mixture of safflower and peanut oils, contained $<0.3 \%$ (weight percentage of total fatty acids) of ALA (18:3 $\omega$-3), DHA's usual dietary precursor, and $54 \%$ linoleic acid $(18: 2 \omega-6)$, the primary dietary $\omega-6$ fatty acid. The $\omega-6: \omega-3$ ratio was 180 ; in addition to the low level of ALA, the high ratio reduces uptake of ALA and synthesis of DHA due to competition between the two fatty acid classes.

The high-ALA diet ( $n=4,2$ male, 2 female), with soybean oil as the sole dietary fat, contained $8 \%$ ALA and 54\% linoleic acid with an $\omega-6: \omega-3$ ratio of 7 . This diet has been shown to support normal levels DHA in the retina and cortical gray matter (Neuringer et al., 1986).

The "DHA" diet ( $n=4,2$ male, 2 female) consisted of a mixture of plant, animal, and fish oils providing the long-chain polyunsaturated fatty acids $0.6 \%$ DHA (22:6 $\omega-3), 0.2 \%$ EPA $(22: 5 \omega-3), 0.2 \%$ arachidonic acid $(20: 4 \omega-6)$, and $1.4 \%$ ALA. This diet was designed to approximate the fatty acid composition of rhesus and human milk and leads to retina and brain levels of DHA similar to the ALA group, although circulating levels of DHA are higher.

The diets contained all essential nutrients at the recommended levels and supported normal growth and health. Animals all were in good health at the time of the MRI scans, as confirmed by daily observation by veterinary staff. Food intake was adjusted to maintain body weights within the normal range and, except for slightly higher weights in the deficient females at ages 2-7 years, weights across the lifespan did not differ significantly among the three diet groups. All aspects of the studies complied with Society for Neuroscience Policies on the Use of Animals and Humans in Neuroscience Research and National Institutes of Health (NIH) guidelines and were approved by the Oregon Health and Science University Institutional Animal Care and Use Committee.

\section{MRI acquisition and processing}

MRI scanning. Imaging was performed during a single session for each animal subject on a 3T Siemens Tim Trio scanner with a 15-channel knee coil adapted for monkey head scanning. Subjects were sedated with an initial dose of ketamine $(5 \mathrm{mg} / \mathrm{kg})$, intubated, and maintained under $1 \%$ isoflurane anesthesia for the duration of the MRI procedures. Initial pilot studies determined that this was the optimal sedation protocol for rsfcMRI scans. Physiological monitoring throughout anesthesia included heart rate, respiration, and peripheral oxygen saturation. Data acquisition included four high-resolution, T2-weighted structural images $\left(\mathrm{TR}=10240 \mathrm{~ms}, \mathrm{TE}=95 \mathrm{~ms} ; \mathrm{FA}=150^{\circ} ; 0.5 \mathrm{~mm}^{2}\right.$ in plane resolution, $1 \mathrm{~mm}$ slice thickness, 56 slices, FOV $=128 \times 128 \mathrm{~mm}$ ), which were averaged to improve signal-to-noise ratio. A functional MRI scan lasting 30 min was then begun exactly $45 \mathrm{~min}$ after the time of ketamine administration (delaying the beginning of the acquisition as necessary) using a gradient echo echoplanar imaging sequence sensitive to blood-oxygenlevel-dependent contrast $\left(\mathrm{TR}=2070 \mathrm{~ms}, \mathrm{TE}=25 \mathrm{~ms}, \mathrm{FA}=90^{\circ}, 1.5\right.$ $\mathrm{mm}^{3}$ voxels, 32 slices with interleaved acquisition, $\mathrm{FOV}=96 \times 96 \mathrm{~mm}$ ). A field map scan was acquired $(\mathrm{TR}=450 \mathrm{~ms}, \mathrm{TE}=5.19 \mathrm{~ms} / 7.65 \mathrm{~ms}$, $\mathrm{FA}=60^{\circ}, 1.25 \times 1.25 \times 2 \mathrm{~mm}^{3}$ voxels, 40 slices, $\left.\mathrm{FOV}=120 \times 120 \mathrm{~mm}\right)$ to correct for image distortion (see Image processing, below).
Image processing. The raw fMRI data underwent standard preprocessing using the FMRIB software library's FEAT preprocessing tools. This included slice-timing correction, rigid-body correction for head motion, unwarping of field map distortions, and rigid-body coregistration of the fMRI volumes with the high-resolution, T2-weighted structural image. The structural image was transformed using 12-paramater affine registration to conform to a T2-weighted atlas image that was an average of 112 monkeys (McLaren et al., 2009). This atlas image was also linearly registered onto the widely used macaque F99 atlas, which is freely available as part of the CARET software package (http://brainvis.wustl. edu/wiki/index.php/Main_Page). Therefore, the registration parameters obtained from each step allowed raw fMRI images to be transformed into F99 space, combining motion correction, field map unwarping, and atlas transformation in one interpolation step. Several additional steps were also taken to prepare the data for connectivity analyses (Fox et al., 2005), including temporal band-pass filtering $(0.009 \mathrm{~Hz}<\mathrm{f}<0.08 \mathrm{~Hz})$, spatial smoothing ( $3 \mathrm{~mm}$ full-width at half-maximum), and regression of nuisance signals. Nuisance signals consisted of the whole-brain signal and the six parameters related to rigid-body motion correction. Nuisance signals were also band-pass filtered before regression (Hallquist et al., 2013).

Motion censoring. All subjects were monitored continuously to confirm effective sedation during scanning. Although no detectable motion was observed for any subject, motion censoring was nonetheless performed according to procedures recommended in prior work (Fair et al., 2012; Power et al., 2012; Iyer et al., 2013).

\section{Connectivity analyses}

Cortical region selection. All cortical regions were predefined with a parcellation of the F99 atlas included in the CARET software as "LVE00," which was based on previous anatomical work (Lewis and Van Essen, 2000). The right primary visual cortex (V1) within this parcellation was used as the seed region for analyzing cortico-subcortical connectivity in the visual pathway (Fig. 1). For analyzing cortico-cortical connectivity (Fig. 3), region Ig-Id of the right dorsal anterior insula was used as the seed. This region was chosen for its predictable pattern of distributed anatomical and functional connectivity in both the human and macaque (Mesulam and Mufson, 1982; Mufson and Mesulam, 1982; Hutchison et al., 2011; Hutchison et al., 2012) and for its role as a higher-order region of multimodal integration in both species. The entire region set was used for community detection analyses, as described in Community detection, below.

Subcortical region selection. A single, bilateral mask of the entire thalamus and superior colliculus was manually traced in the macaque F99 standard atlas space. The combined MRI and histological atlas provided by Saleem and Logothetis (2012), which provides detailed boundaries for subcortical structures in the F99 atlas, was used as a visual reference for delineating boundaries of the thalamus and superior colliculus in the F99 T1-weighted image volume, which was in register with our T2-weighted atlas, as described previously.

Connectivity. Time series were computed for cortical regions of interest (ROIs) by averaging signal intensity across voxels within a given ROI at each time point. For seed-region-based analyses (Figs. 1, 3), crosscorrelations $(-1<\mathrm{r}<1)$ were then computed between the ROI and all other voxels in the brain. This generated a whole-brain correlation map for each subject. Within each group, voxelwise connectivity maps were generated using a second-level fixed effects analysis using the $4 \mathrm{dfp}$ suite (ftp://ftp.imaging.wustl.edu/pub/raichlab/4dfp_tools/). Between-group comparisons were performed using two-tailed rank-sum tests (Wilcoxon exact). To account for multiple comparisons, Monte Carlo simulations were run using the AlphaSim tool included in AFNI (http://afni.nimh. nih.gov/afni/). A threshold of 65 contiguous voxels with a $|Z|>2$ (fixed effects) or $p<0.05$ (rank-sum comparisons) was used to obtain clusters with $\alpha<0.05$. In analyzing cortico-subcortical connectivity (Fig. 1), the tracing of the thalamus and superior colliculus was used as a mask, only showing correlations of voxels within its boundaries to V1. Connectivity maps for seed region Ig-Id (Fig. 3) display results within all cortical gray matter. To test for differences in ROI-ROI connectivity (Figs. 2, 4), the main effect of group was first assessed (Kruskal-Willis) and then pairwise 
comparisons (Wilcoxon exact, two-tailed) were only performed where there was a significant main effect $(p<0.05)$.

\section{Community detection}

To assess community organization in the cortex, cross-correlations were first computed between all cortical ROI pairs, yielding a correlation matrix of size $176 \times 176$ for each subject. Individual subject matrices were then averaged together within each group, yielding a single adjacency matrix for each group. To combine correlation coefficients $(r)$ across subjects within each group, the Schmidt-Hunter method for metaanalyses of $r$ values was used because it is more conservative than comparable methods (Field, 2001; Salvador et al., 2005; Dosenbach et al., 2007; Fair et al., 2009; Power et al., 2011). To further the validity of graph-theoretical analyses, connections between neighboring ROIs (ROIs sharing a border with one or more voxels) were excluded. This approach is outlined by previous work (Power et al., 2011) demonstrating that functional connectivity data may be biased by short range, nonbiological influences such as partial voluming and the spatial blurring typically applied during preprocessing. In addition, edges used to define the adjacency matrix are specified as the subset of the connections with the highest magnitude $r$ values. The percentage of connections selected from among the total number of possible connections possible is termed the connection density. To provide a broad survey of the solution space, analyses depicted in Figure 5 were performed using $4 \%$ and 10\% connection densities in each group; that is, edges were considered to exist for only connections within the highest $4 \%$ or $10 \%$ of $r$ values.

The resulting group matrices were examined using the community detection algorithm for undirected, weighted matrices adapted from Newman (2006) and available at https://sites.google.com/site/bctnet/. This algorithm iteratively subdivides a given adjacency matrix into nonoverlapping groups of nodes (communities) in a way that maximizes the quality index $(Q)$. Q measures the fraction of edges within community partitions minus the expected fraction of such edges in an equivalent network where edges are rewired at random. Therefore, $Q$ represents the overall segregation between identified communities. It is defined formally as follows:

$$
Q=\frac{1}{4 m} \sum_{i j}\left(a_{i j}-\frac{k_{i} k_{j}}{2 m}\right) \delta\left(c_{i}, c_{j}\right)
$$

where $m$ is the total number of nodes in the network; $k_{i}$ and $k_{j}$ are the degree of nodes $i$ and $j$, respectively; $c_{i}$ is the community to which node $i$ belongs; and $\delta\left(c_{i}, c_{j}\right)$ is the delta function where $\delta\left(c_{i}, c_{j}\right)=1$ if $c_{i}=c_{j}$ and 0 otherwise.

It is well known that the Newman algorithm, and modularity algorithms in general, suffer from inconsistent partitions. To generate a single, stable depiction of whole-brain community structure (individual parcellations in Fig. 5), we used the method of maximum within-module connectivity likelihood (Rubinov and Sporns, 2011; Liang et al., 2012). This proceeds as follows. First, we iterated the Newman algorithm on a given adjacency matrix 50 times to generate a distribution of network partitions. Then, for each edge in the adjacency matrix, we measured how often it was that both nodes were within the same module across network partitions. The within-module likelihood matrix was generated by assigning a 1 to all edges contained within the same module at least $75 \%$ of the time, and assigning a 0 to all others. The final community structure was generated from this matrix.

\section{Statistical comparison of community structure}

To assess the significance of differences in the modular organization between DHA-fed and deficient groups (Fig. 4a), the normalized mutual information (MI) between the community structures was obtained as described by Meila (2007). We then performed standard permutation testing (Bassett et al., 2008) to determine whether the MI was lower than what would be expected by chance. First, a null distribution of MI values was built by randomizing the group labels of individual subjects, redoing analyses, and recomputing MI on the randomized groups. For a comparison between DHA $(n=4)$ and deficient $(n=7)$ groups, this yields a total of 330 distinct permutations. A $p$-value was then calculated as the percentage of null MI values that were below the observed MI. This process was repeated 50 times to obtain an average $p$-value. To demonstrate the robustness of this approach, MI and corresponding $p$-values were obtained at a range of connection densities between $2 \%$ and $10 \%$. This is consistent with the range of connection densities used in previous work characterizing whole-brain community structure (Power et al., 2011). This method was also used to test differences between ALA-fed and deficient animals (not significant, data not shown), but not for DHA versus ALA because the number of permutations (70) was deemed too low.

The quality indices $(Q)$ of DHA-fed and deficient group matrices were also compared using permutation testing (Fig. 4b). The observed between-group difference in $Q$ was obtained at each connection density and compared with the null distribution of $Q$ differences (in absolute values) obtained on the 330 randomized groups. A $p$-value (two-tailed) was then calculated as the percentage of null $Q$ differences that exceeded the observed difference.

Individual patterns of modular organization (Fig. $4 c$ ) were assessed by performing community detection separately on each subject based on $4 \%$ connection densities. MI was then calculated between all possible pairs of subjects. To visualize between-subject similarities, the pairwise MIs were treated as a similarity matrix and input into the MATLAB function "mdscale" (http://www.mathworks.com/help/stats/mdscale. $\mathrm{html})$. This function provides nonmetric 2D scaling to approximate Euclidean distances between points, where the ordering of distances attempts to maintain the order of MI. Therefore, if A and B have greater MI than C and D, then the same should hold in the embedding. Statistical comparison of DHA-fed and deficient subjects was performed using pairwise MIs. We considered the following test statistic, (average withingroup MI) - (average between-group MI), as an indicator of group differences. Standard permutation testing was then performed with the 330 randomized groups to obtain a two-tailed $p$-value, given as the percentage of null test statistics (in absolute values) that exceeded the observed statistic.

\section{Results}

\section{Visual pathway connectivity}

The first set of analyses examined functional connectivity within the major pathways from the retina to the primary visual cortex (area V1). In the animals consuming DHA, as expected and consistent with prior human fcMRI work (Zhang et al., 2010; Fair et al., 2010), these pathways are reflected in strong connectivity of the major visual subcortical relay nuclei-the LGN and the SCwith V1 (Fig. 1). In contrast, V1-LGN and V1-SC connectivity was absent in the deficient group. Between-group comparisons confirmed that the deficient animals had significantly reduced connectivity in both of these pathways relative to the DHA group (Fig. 1). In ALA-fed animals, connectivity strength was intermediate. In addition, we examined the connectivity between right and left hemispheres in V1, where homotopic rs-fc is thought to reflect the interaction with subcortical hubs that mediate interhemispheric connectivity (Savazzi et al., 2007; Uddin et al., 2008). We also examined similar homotopic interactions in primary somatosensory cortex (S1) for comparison. Deficient animals had decreased connectivity relative to DHA-fed animals in V1, but not in S1 (Fig. 2), and the ALA group was again intermediate. Thus, the homotopic connectivity of V1 and S1 were not equally affected by dietary manipulation, perhaps due to a specific role of DHA in visual sensory transduction and transmission. Together, these results provide strong evidence of the role of dietary DHA in strengthening the connectivity of early visual pathways and demonstrate that rs-fcMRI is sensitive to these changes.

\section{Cortico-cortical connectivity of higher-order regions}

To determine whether group differences are also observable in the organization of large-scale systems within the cerebral cortex, we examined network organization using the parcellation scheme introduced by Lewis and Van Essen (2000) consisting of 
176 cortical ROIs (see Materials and Methods). We first tested the hypothesis that distributed connectivity between higher-order association cortical regions would be disrupted in $\omega$-3FA-deficient animals by examining connectivity with an individual seed region (area Ig-Id) in the dorsal anterior insula. The dorsal anterior insula is a higher-order region of multimodal integration in the macaque (Mesulam and Mufson, 1982; Augustine, 1996) and a key hub of the "cinguloopercular" cognitive control network in humans (Dosenbach et al., 2007; Fair et al., 2007). Insular anatomical and functional connectivity in the macaque is well studied and mirrors human rs-fcMRI work. Its connections encompass orbitofrontal, lateral prefrontal, and nearby temporal cortex, as well as the cingulate gyrus (Mesulam and Mufson, 1982; Mufson and Mesulam, 1982; Hutchison et al., 2011; Hutchison and Everling, 2012). Our connectivity maps (Fig. 3) were consistent with these reports and further revealed widespread and distinct connectivity differences between DHA-fed and deficient animals. In between-group comparisons, the deficient group showed decreased positive correlations with multiple higherorder associational areas, including the cingulate, ventromedial prefrontal, orbitofrontal, dorsolateral prefrontal, superior temporal sulcus, and medial parietal areas, and decreased negative correlations with primary visual and somatosensory cortices. Differences between the ALA-fed and deficient groups were not statistically significant, suggesting that the effects were largely specific to dietary DHA. Analysis of homotopic connectivity within distinct areas of higher-order association cortex confirmed that many, but not all, areas showed increased connectivity as a result of DHA consumption (Fig. 4), including prefrontal, insular, and temporal cortex.

\section{Whole-brain modular organization}

Next, we assessed large-scale modular organization by applying community detection to the region-to-region connectivity matrices encompassing all possible ROI pairs. This approach was based on work by Newman (2006) and the overall strength of our modularity assignments was quantified by the quality index $Q$. Community detection at each connection density demonstrated a combination of bilaterally symmetric distributed networks and local sensory/motor networks (Fig. 5). Both also contained one or two small, provincial networks in higher-order areas, although such networks comprised very few regions overall.

Qualitative comparisons of the community maps revealed several important distinctions between experimental cohorts. In animals fed DHA, at the $4 \%$ density, 4 prominent, relatively well distributed, bilateral functional networks were identified (Fig. 5, green, yellow, pink, and red colors). These networks bore a qualitatively striking, although not identical, relationship to the major cognitive networks typically identified in the human rs-fcMRI literature (Power et al., 2011; Yeo et al., 2011). We can loosely
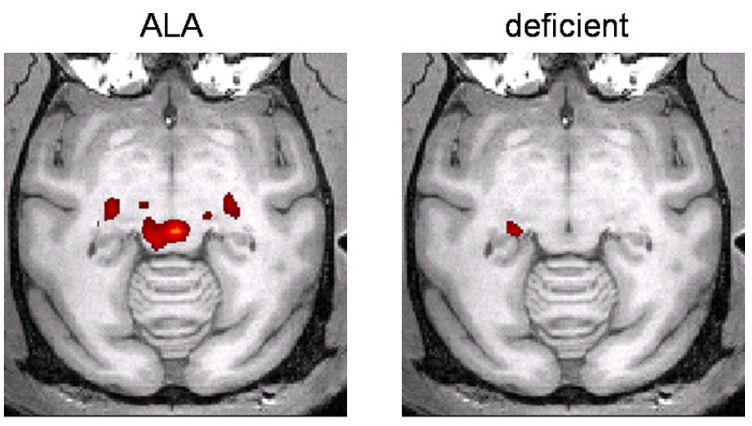

$\mathbf{Z}$
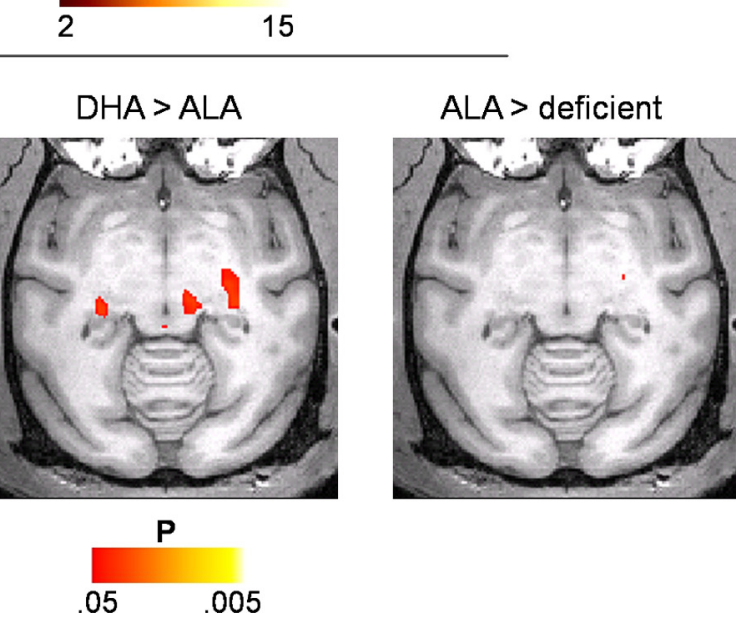

.05

005

Figure 1. Connectivity with primary visual cortex in the LGN and SC is reduced in animals without dietary DHA. Top, Connectivity maps $(\mid 2>2)$ within thalamus and superior colliculus show connectivity strength from V1 to LGN and SC in the order of DHA $>$ ALA $>$ deficient. Bottom, Pairwise group comparisons revealed significant ( $p<0.05$, Wilcoxon exact, two-tailed) differences between the DHA-fed and deficient groups (left) and between the DHA and ALA groups (middle). No significant differences were observed between the ALA-fed and deficient groups (right).

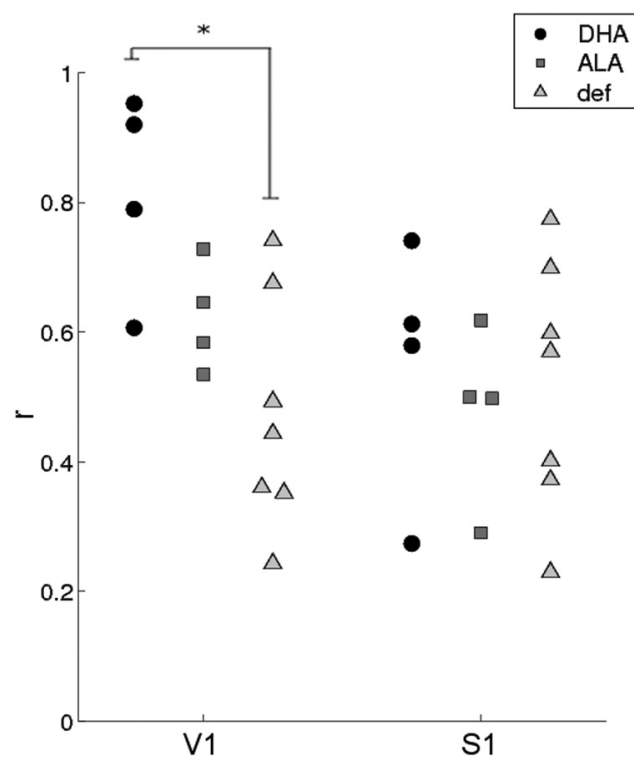

Figure 2. Homotopic connectivity in V1 and in S1. Correlation values (Pearson's $r$ ) were assessed between corresponding right and left regions in primary visual cortex (V1) and primary somatosensory cortex (S1). Results demonstrate that deficient animals have decreased homotopic connectivity in V1 ( ${ }^{*} p<0.03$, Wilcoxon exact, two-tailed), but not in S1 cortex. A significant main effect of group was confirmed for V1 $(p<0.04)$, but not for S1 ( $p>0.8)$. Homotopic connectivity in V1 was intermediate in ALA animals, although this was not significant. 

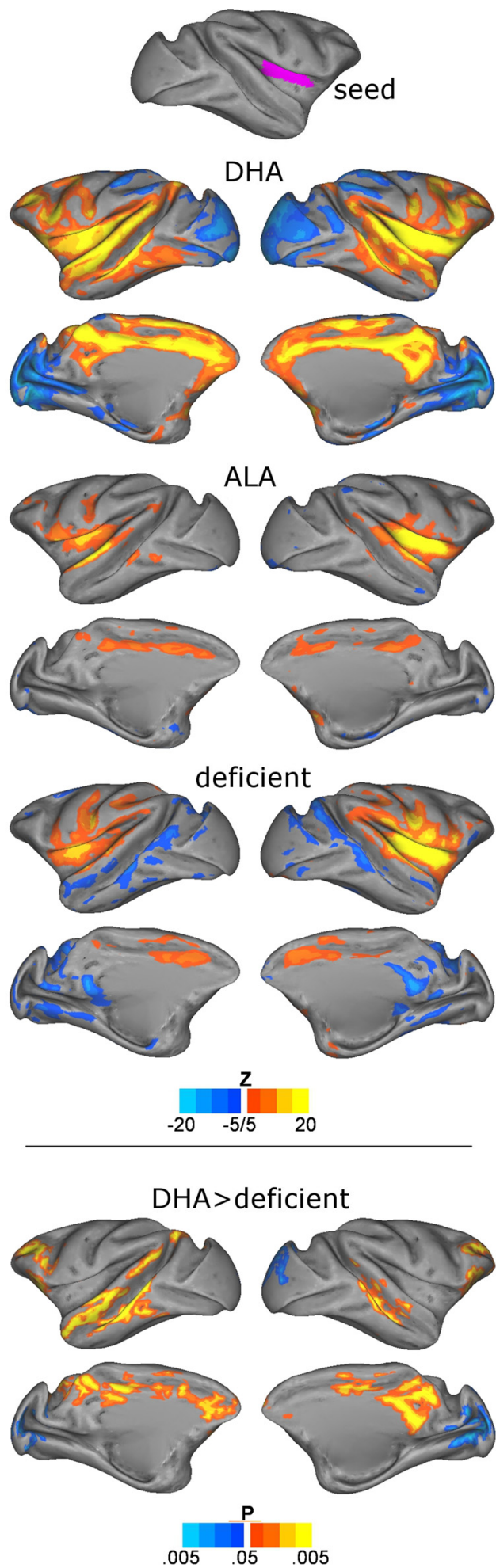

Figure 3. Connectivity between association cortices is greater in the DHA group. Seed-based connectivity maps for dorsal anterior insula (region Ig-ld) show widespread and distinct connectivity patterns in DHA-consuming animals relative to the deficient group. Top, Seed region. Middle, Connectivity maps displayed within the DHA, ALA, and deficient groups $(|Z|>5)$. Bottom, Comparison of DHA-fed and deficient groups revealing greater positive connectivity among multiple higher-order associational cortical areas and greater segregation (negative correlations) of these units from primary sensory cortex in the DHA group. No significant clusters were observed when comparing DHA versus ALA or ALA versus deficient. classify these based on their present anatomical structure and their current naming conventions in the human literature as the dorsal attention (green), frontoparietal (yellow), cinguloopercular (pink), and default mode (red) networks. In addition, we can identify distinct sensory and motor components-early visual (blue) and somatomotor (aqua) — that have shown marked similarities across species in previous studies (Vincent et al., 2007; Hutchison and Everling, 2012). In contrast, network partitions in the deficient animals (Fig. 5, bottom left) were quite different. Qualitatively, networks were anatomically more local and, in some cases, comprised contiguous regions within well defined anatomical boundaries. For example, the medial prefrontal to posterior cingulate connection that traditionally has been referred to as the core component of the default system is absent in the deficient animals (Fig. 5, red arrows). Likewise, components for the cingulo-opercular network are also disjointed (Fig. 5, pink arrows). This suggests reduced connectivity among distributed systems in deficient animals, which show only weakly distributed frontoparietal and dorsal attention systems. The ALA group was intermediate and appeared to better recapitulate some of the distributed networks present in the DHA group.

Networks obtained at the 10\% density (Fig. 5, right) illustrate the brain organization patterns that arise when many weaker connections are included. Across all groups and consistent with prior human work (Fair et al., 2009; Power et al., 2011), some networks present at the lower density appeared to merge at this higher density, leading to more locally consolidated networks. However, many distributed systems remained and qualitative differences between groups were still noticeable. In the DHA group, the presence of the dorsal attention, default mode, and cingulo-opercular networks were observed, although the frontoparietal network was no longer detected. In contrast, in the deficient group, only one distributed component remained (presumably related to visual function), whereas other networks present at the $4 \%$ density (frontoparietal and dorsal attention systems) were further degraded and merged with nearby sensory systems. Again, the ALA group appeared intermediate with regard to the presence of distributed networks.

Direct comparison of the MI between the DHA-fed and deficient groups confirmed that differences in modular organization were statistically significant $(p<0.05)$ at connection densities between $3.5 \%$ and $6.5 \%$ (Fig. 6a). Interestingly, the $Q$ values differed widely across these connection densities, with the deficient group showing significantly greater $Q$ values (Fig. $6 b$ ). This indicates increased segregation between the identified communities in the deficient group relative to the DHA-fed group. Comparisons of ALA-fed and deficient animals did not show differences with these metrics (data not shown). In addition, community detection performed at the single-subject level revealed separate clusters of subjects corresponding to group assignments (Fig. 6c), highlighting the similarities of individual animals within each group. Together, these data provide strong evidence that deficient animals have distinct patterns of cortical organization relative to DHA-fed animals. Furthermore, our findings suggest that DHA consumption is important for the development and/or maintenance of distributed, large-scale systems.

\section{Discussion}

Our results demonstrate an effect of life-long $\omega$-3FA deficiency on brain functional organization, including connectivity within the visual system and, more broadly, the large-scale network topology of the brain. We first showed that $\omega$-3FA deficiency sig- 


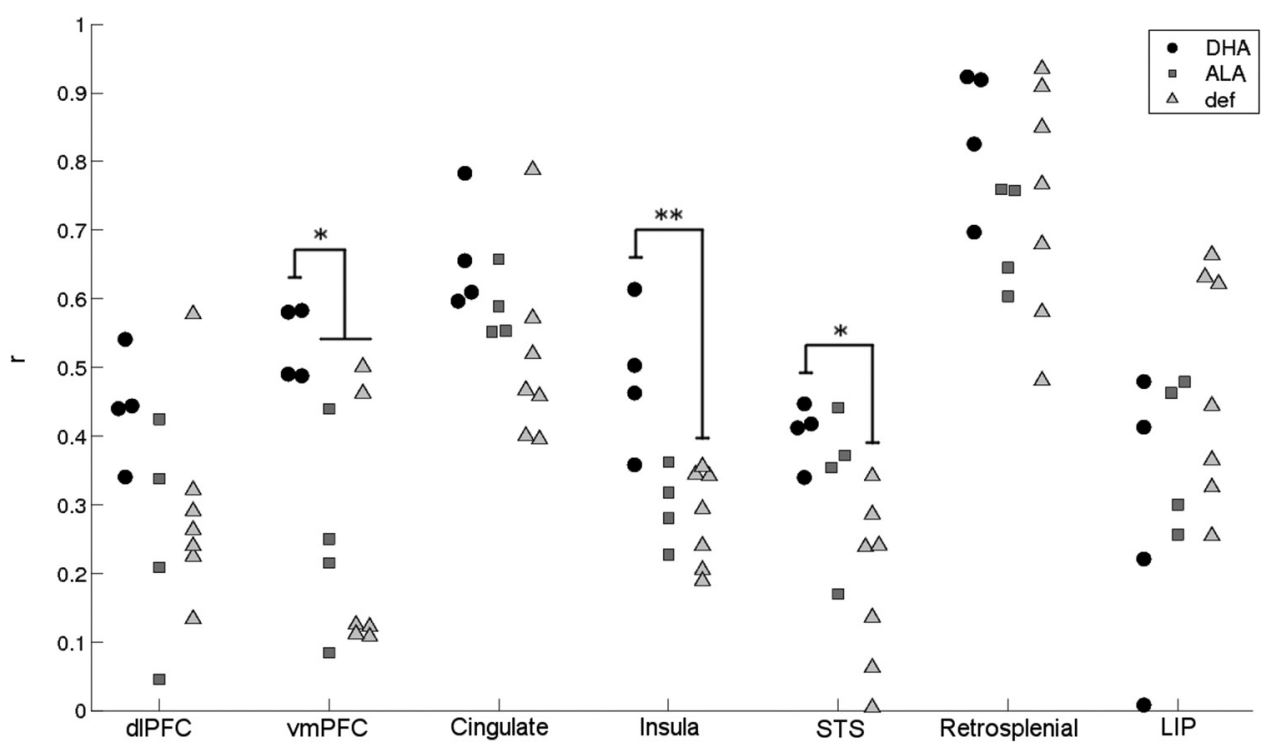

Figure 4. Homotopic connectivity of higher-order association cortex is greater in the DHA group. Correlation values were assessed between corresponding right and left regions within distinct anatomical areas of higher-order, polymodal associational cortex. Areas that showed a significant main effect of group included ventromedial prefrontal (vmPFC), insula cortex, and the superior temporal sulcus (STS) $(p<0.04, p<0.03, p<0.03$, respectively; Kruskal-Willis). Pairwise comparisons (Wilcoxon exact, two-tailed) within these areas confirmed increased connectivity in DHA-fed animals relative to deficient and/or ALA-fed animals $\left({ }^{*} p<0.05 ;{ }^{* *} p<0.01\right.$ ). Regions from the LVE00 parcellation within each anatomical area were as follows: dlPFC (dorsolateral prefrontal cortex), 45, 46 p/v; vmPFC (ventromedial prefrontal cortex), 10 m, 12, 14 r; cingulate, 23, 24a/b, 31, 32; insula, Ig/ld, Pi, Ri; STS (superior temporal sulcus), TP0i, TPOr, FST, IPa; retrosplenial, MDP; LIP (lateral inferior parietal), LIPd, LIPv. For subgroups with more than one region, correlation values were averaged across regions within each subject.

nificantly reduced connectivity from the primary visual cortex to its two major retinocortical relay nuclei (the LGN and SC). Likewise, $\omega$-3FA deficiency reduced homotopic V1 connectivity (Fig. 2 ), which may reflect reduced connectivity with subcortical targets (Savazzi et al., 2007; Uddin et al., 2008; O'Reilly et al., 2013). Last, $\omega$-3FA deficiency appeared to affect large-scale network structure as measured via community detection.

\section{$\omega$-3FA deficiency and the visual system}

Although recent studies have characterized effects of $\omega$-3FA deficiency on retinal projections in rodents (de Velasco et al., 2012), effects on cortico-subcortical connectivity within the visual system have not been identified previously, nor have there been direct assessments of effects on functional connectivity within this pathway. Interestingly, the lack of significant LGN-V1 connectivity in non-DHA-consuming animals suggests that positive rs-fc within this pathway during the anesthetized state does not necessarily coincide with the existence of awake visual function. Therefore, the lack of synchrony at the coarse spatial and temporal resolution afforded by rs-fcMRI may signify atypical function, but not necessarily lack of function.

In addition, connectivity within the visual system was greater in ALA-consuming animals than in the deficient animals, but reduced relative to the DHA-consuming animals (Figs. 1, 2). It has similarly been shown that feeding human infants with ALA but no DHA results in lower levels of cerebral DHA accretion than in breastfed infants (Guesnet and Alessandri, 2011). Therefore, our results support previous work indicating that the use of ALA as the only source of $\omega$-3FAs may be insufficient and extend our understanding of the detrimental effects of $\omega$-3FA deficiency on visual system function and the positive functional effects of DHA supplementation (Neuringer et al., 1986; SanGiovanni et al., 2000; O'Connor et al., 2001; Bazan, 2006).

Importantly, the present study is largely consistent with prior work on these animals. We have previously documented effects on retinal function, visual acuity development, and visual atten- tion in $\omega$-3FA-deficient monkeys. Electroretinographic studies of retinal function in these same monkeys showed a delay in rod photoresponse recovery that arose in infancy and persisted into adulthood and a reduction in rod sensitivity (an increase in the flash intensity needed to evoke a half-maximum-amplitude response) that emerged in adulthood (Jeffrey et al., 2002; Jeffrey and Neuringer, 2009). We note that the ALA and DHA groups did not significantly differ in any electroretinographic measures, although the present connectivity measures appear to show the ALA group as intermediate. In addition, our earlier studies of $\omega$-3FA-deficient monkeys showed a delay in visual acuity development during infancy (Neuringer et al., 1986) and several studies of human infants have reported faster acuity development in infants fed formulas with added DHA (Birch et al., 1998, 2010; SanGiovanni et al., 2000; O’Connor et al., 2001).

\section{$\omega$-3FA deficiency and network structure}

Several studies have also shown that $\omega$-3FA deficiency leads to various cognitive deficits (Moriguchi et al., 2000; Wainwright, 2002; Hoffman et al., 2009; McNamara et al., 2010; Ryan et al., 2010). Therefore, we hypothesized that differences between groups would be observable in distributed links between higherorder cortical areas. Our findings indeed suggest that dietary $\omega$-3FA deficiency alters function beyond the visual system in a specific, yet widespread manner across the cerebral cortex. In the DHA group, multiple distributed areas of higher-order associational cortex showed significantly increased positive connectivity and increased segregation of these units from primary sensory domains relative to the deficient group (Figs. 3, 4). This pattern parallels the functional organization of the homologous association cortical regions in the human brain. In humans, there is strong linkage among nonsensory, distributed systems involved in cognitive control, higher-order processing, and executive function including the cingulo-opercular, default mode, and frontoparietal networks (Dosenbach et al., 2007; Stevens et al., 2012). Accordingly, the color scheme we used in Figure 5 is based 


\section{$4 \%$ density}
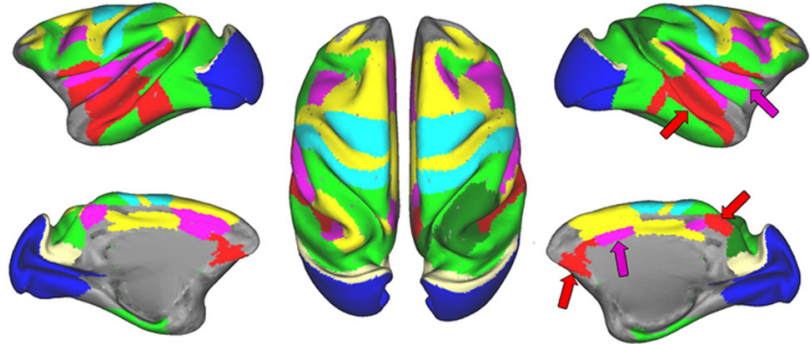

DHA
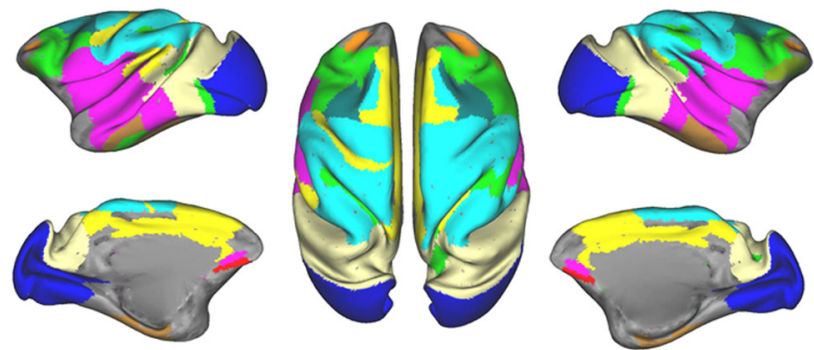

ALA
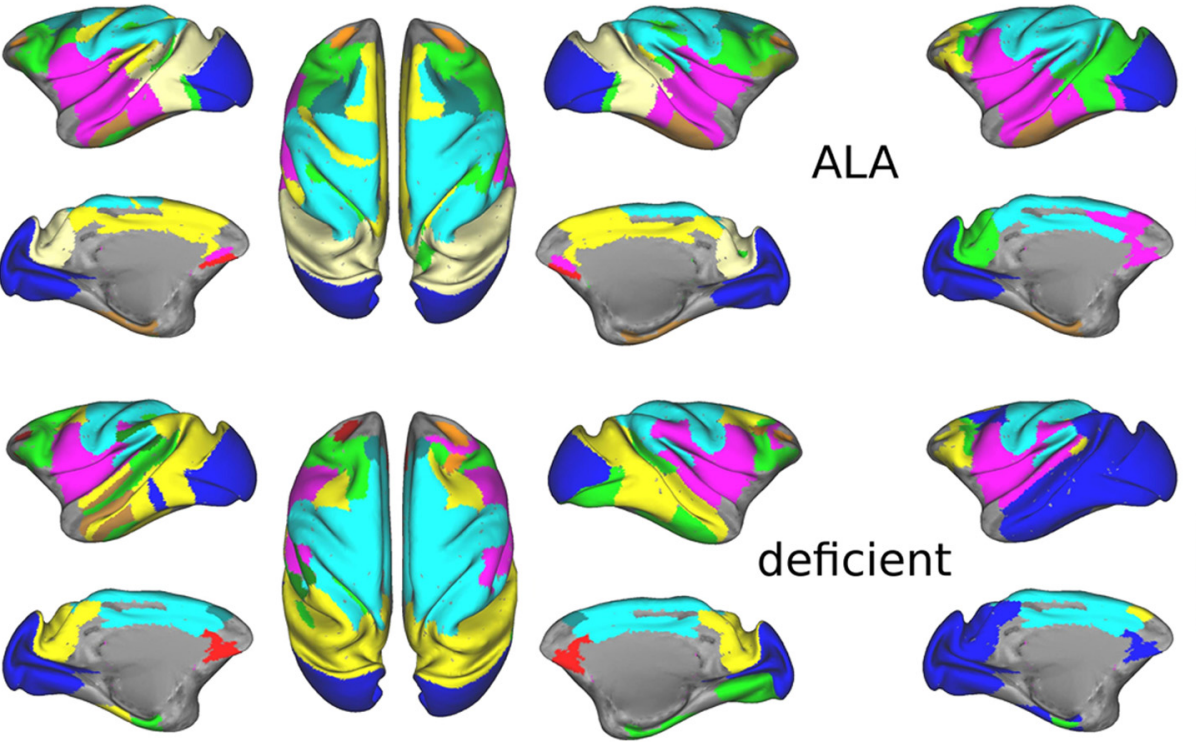

\section{0\% density}
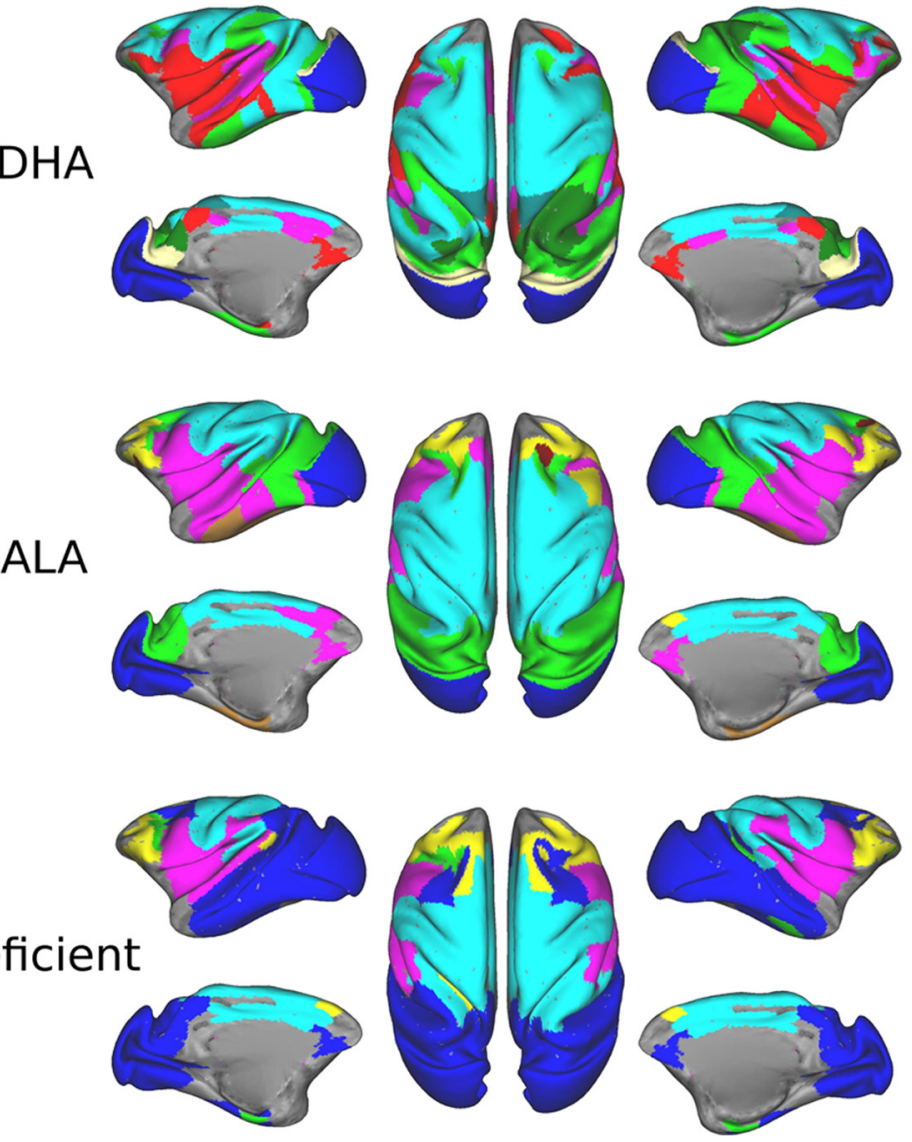

Figure 5. Whole-brain functional community structure is atypical in $\omega$-3FA-deficient animals. Color-coded functional communities as defined on the "LVE00" parcellation are presented for each cohort of monkeys (rows) at $4 \%$ and $10 \%$ connection densities (left and right columns, respectively). Across both densities, functional communities in the DHA group exhibit at least three distributed systems within association cortex loosely classified here as default mode (red), cingulo-opercular (pink), and dorsal attention (green), in addition to early sensorimotor (aqua) and visual systems (blue). Results for the deficient cohort reveal more localized communities and a notable weakness or absence of these distributed systems. Community structure for ALA-fed animals was intermediate. Looking across densities, one can observe within all groups that some communities present at $4 \%$ appear to merge at $10 \%$. Other colors were also assigned based on anatomical location as follows: frontoparietal (yellow), anterior prefrontal (yellow/orange), extrastriate occipital (cream), inferior temporal/parahippocampal (brown), premotor (gray blue), parietal (dark green), and other (dark red). Gray cortex is not covered by the LVE00 parcellation.

a

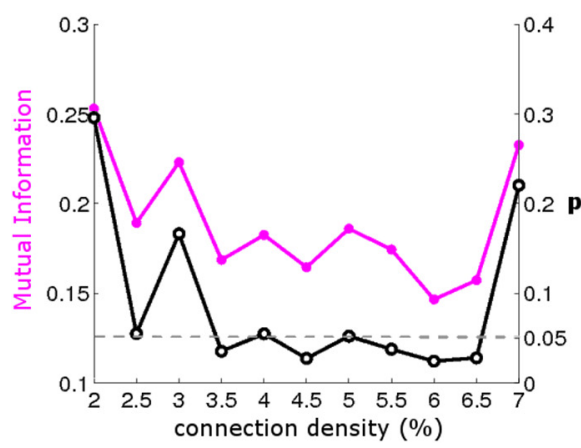

b

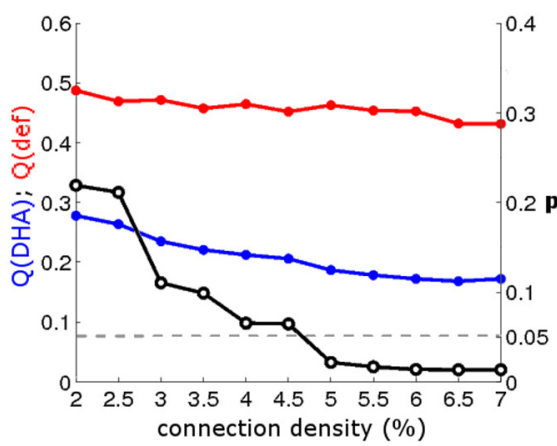

C

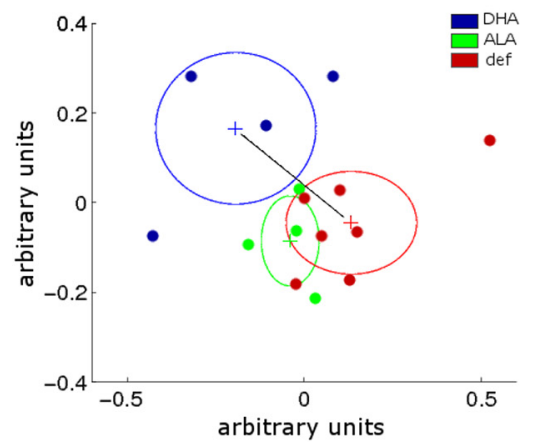

Figure 6. Statistical comparisons confirm distinct patterns of modular brain organization between DHA-fed and deficient groups. $\boldsymbol{a}$, Mutual information between the DHA-fed and deficient groups' community structure (magenta line; left $y$-axis) is plotted at connection densities from $2 \%$ to $7 \%$. Statistical significance of observed MI (black line; right $y$-axis) considered whether observed MI was below what would be expected by chance (dotted line indicates $p=0.05$ level, permutation testing). $\boldsymbol{b}, Q$ values were calculated for the DHA-fed and deficient groups separately (blue and red lines, respectively; left $y$-axis). Statistical significance of differences in $Q$ (black line; right $y$-axis) were calculated using two-tailed permutation testing. c, Embedding diagram of similarities in single-subject community structure. Individual subjects are plotted as dots such that proximity between dots reflects the ordering of the magnitude of Ml between subjects. Distinct clusters corresponding to group assignments are observable. Ellipses of variance are overlaid with the center marked ( + ) where the radius of each axis corresponds to 1 SD. Statistical comparison of DHA-fed and deficient subjects revealed significantly greater within-group MI than between-group MI ( $p<$ 0.02 , permutation testing, two-tailed; black line for visualization). 
heavily on current rs-fcMRI literature in humans, and to a lesser extent in macaques, and should be regarded as a theoretical guide here. Future task-related work in awake animals will help to better characterize the role of functional brain networks in the macaque (Hutchison and Everling, 2012). We also note that, in the typical human brain, these networks tend to exhibit a higher degree of segregation from one another, as measured with $Q$ (Fair et al., 2009; Power et al., 2011), than what was seen in the DHAfed monkeys. Nonetheless, the whole-brain topology of DHA-fed animals revealed distributed networks that were markedly similar to those identified in the typical adult human brain, whereas these networks were considerably less defined in deficient and ALA-fed animals (Fig. 5).

Community structure in DHA-fed and deficient groups showed statistical differences across a variety of graph theoretical measures (Fig. 6), indicating that differences were meaningful and robust among sparse connectivity matrices. Work related to how these changes correspond directly to complex behaviors (Bassett et al., 2011; Stevens et al., 2012) in this model will be an important area of study in future work. With that said, we highlight that prior work in these animals has shown differences in visual attention, specifically, fixation patterns that may reflect slower visual processing, in $\omega$-3FA-deficient monkeys during infancy (Reisbick et al., 1997). Similar effects have been reported in human infants as a function of DHA intake (Carlson and Werkman, 1996; Colombo et al., 2004). It is notable that our previous monkey studies found deficits in retinal function and acuity development in the deficient group but not between the ALA and DHA groups, a different pattern than found here in measures of connectivity and cortical organization; however, as noted throughout the Discussion, a number of human studies have demonstrated effects of DHA supplementation on visual development and cognitive function. Our results indicate that the ALA animals are generally intermediate to the DHA-fed and deficient groups and therefore appear to provide evidence that functional connectivity is sensitive to both total $\omega$-3FA deficiency and DHA supplementation.

\section{Relationship of DHA to development and disorders}

The results of this study cannot determine whether the observed effects on connectivity are due to atypical maturation, maintained deficiency across the lifespan, or accelerated aging. However, several lines of evidence support the existence of developmental effects. First, dysregulation of the putative networks identified here as being susceptible to fatty acid diet manipulation have been related to several neurodevelopmental disorders (Fair et al., 2012) and increasing attention has been paid to the possible beneficial role of DHA (Freeman et al., 2006). Children with attentiondeficit hyperactivity disorder (ADHD) have shown low blood levels of DHA and other essential fatty acids (for review, see Raz and Gabis (2009)), whereas $\omega$-3FA deficiency in rodents and juvenile nonhuman primates has been reported to increase activity and stereotypy consistent with an ADHD-like behavioral pattern (Reisbick et al., 1994; Levant et al., 2010). On a cellular level, low levels of $\omega$-3FA during prenatal and postnatal development lead to changes in neurotransmitter metabolism, particularly in brain areas such as the prefrontal cortex that underlie complex cognitive functions (Delion et al., 1996; Ahmad et al., 2008). The period of "exuberant" synaptogenesis occurring in the first two postnatal years demands the incorporation of large amounts of DHA into synaptic membranes and corresponds with a surge in brain DHA accretion (Martinez, 1992). Deficiency during this period may have the most detrimental and long-term effects for brain function (Innis, 2007). For example, strong effects have been observed on the fatty acid composition of neural growth cones (Auestad and Innis, 2000) and on the number and complexity of synaptic contacts (Tixier-Vidal et al., 1986; Wainwright, 2002; He et al., 2009). Therefore, lower brain DHA critically affects brain development and may lead to reduced connectivity within the early visual pathway and higher-order cortical pathways.

\section{Limitations}

Although our findings are concordant with prior work in this cohort and with current literature on $\omega$-3FA deficiency, the small sample sizes of this study remain an important limitation, constrained by the number of animals available on this long-term protocol. To account for these concerns, we used highly conservative statistical methods for assessing all group differences via nonparametric testing. Although differences were strong and consistent even with these constraints, confirmation with random effects modeling will require the generation of larger groups of monkeys on similar strictly controlled diets for many years.

\section{Conclusions}

The present work demonstrates a detrimental effect of lifelong dietary $\omega$-3FA deficiency upon multiple aspects of brain functional connectivity. We have shown that dietary DHA is crucial for visual pathway connectivity and large-scale brain organization. Future work will be able to better distinguish the impacts of $\omega$-3FA deficiency from those of DHA augmentation in the context of normal $\omega$-3FA consumption. In addition, a longitudinal approach will be necessary to characterize effects during development, throughout the lifespan, or as a function of aging.

\section{References}

Ahmad SO, Park JH, Radel JD, Levant B (2008) Reduced numbers of dopamine neurons in the substantia nigra pars compacta and ventral tegmental area of rats fed an n-3 polyunsaturated fatty acid-deficient diet: a stereological study. Neurosci Lett 438:303-307. CrossRef Medline

Auestad N, Innis SM (2000) Dietary n-3 fatty acid restriction during gestation in rats: neuronal cell body and growth-cone fatty acids. Am J Clin Nutr 71:312S-314S. Medline

Augustine JR (1996) Circuitry and functional aspects of the insular lobe in primates including humans. Brain Res Brain Res Rev 22:229-244. CrossRef Medline

Bassett DS, Bullmore E, Verchinski BA, Mattay VS, Weinberger DR, MeyerLindenberg A (2008) Hierarchical organization of human cortical networks in health and schizophrenia. J Neurosci 28:9239-9248. CrossRef Medline

Bassett DS, Wymbs NF, Porter MA, Mucha PJ, Carlson JM, Grafton ST (2011) Dynamic reconfiguration of human brain networks during learning. Proc Natl Acad Sci U S A 108:7641-7646. CrossRef Medline

Bazan NG (2006) Cell survival matters: docosahexaenoic acid signaling, neuroprotection and photoreceptors. Trends Neurosci 29:263-271. CrossRef Medline

Birch EE, Hoffman DR, Uauy R, Birch DG, Prestidge C (1998) Visual acuity and the essentiality of docosahexaenoic acid and arachidonic acid in the diet of term infants. Pediatr Res 44:201-209. CrossRef Medline

Birch EE, Carlson SE, Hoffman DR, Fitzgerald-Gustafson KM, Fu VL, Drover JR, Castañeda YS, Minns L, Wheaton DK, Mundy D, Marunycz J, Diersen-Schade DA (2010) The DIAMOND (DHA Intake And Measurement Of Neural Development) Study: a double-masked, randomized controlled clinical trial of the maturation of infant visual acuity as a function of the dietary level of docosahexaenoic acid. Am J Clin Nutr 91:848 859. CrossRef Medline

Carlson SE, Werkman SH (1996) A randomized trial of visual attention of 
preterm infants fed docosahexaenoic acid until two months. Lipids 31 : 85-90. Medline

Colombo J, Kannass KN, Shaddy DJ, Kundurthi S, Maikranz JM, Anderson CJ, Blaga OM, Carlson SE (2004) Maternal DHA and the development of attention in infancy and toddlerhood. Child Dev 75:1254-1267. CrossRef Medline

Delion S, Chalon S, Guilloteau D, Besnard JC, Durand G (1996) alphaLinolenic acid dietary deficiency alters age-related changes of dopaminergic and serotoninergic neurotransmission in the rat frontal cortex. J Neurochem 66:1582-1591. CrossRef Medline

de Velasco PC, Mendonça HR, Borba JM, Andrade da Costa BL, Guedes RC, Navarro DM, Santos GK, Faria-Melibeu Ada C, Campello Costa P, Serfaty CA (2012) Nutritional restriction of omega-3 fatty acids alters topographical fine tuning and leads to a delay in the critical period in the rodent visual system. Exp Neurol 234:220-229. CrossRef Medline

Dosenbach NU, Fair DA, Miezin FM, Cohen AL, Wenger KK, Dosenbach RA, Fox MD, Snyder AZ, Vincent JL, Raichle ME, Schlaggar BL, Petersen SE (2007) Distinct brain networks for adaptive and stable task control in humans. Proc Natl Acad Sci U S A 104:11073-11078. CrossRef Medline

Fair DA, Nigg JT, Iyer S, Bathula D, Mills KL, Dosenbach NU, Schlaggar BL, Mennes M, Gutman D, Bangaru S, Buitelaar JK, Dickstein DP, Di Martino A, Kennedy DN, Kelly C, Luna B, Schweitzer JB, Velanova K, Wang YF, Mostofsky S, Castellanos FX, Milham MP (2012) Distinct neural signatures detected for ADHD subtypes after controlling for micromovements in resting state functional connectivity MRI data. Front Syst Neurosci 6:80. CrossRef Medline

Fair DA, Dosenbach NU, Church JA, Cohen AL, Brahmbhatt S, Miezin FM, Barch DM, Raichle ME, Petersen SE, Schlaggar BL (2007) Development of distinct control networks through segregation and integration. Proc Natl Acad Sci U S A 104:13507-13512. CrossRef Medline

Fair DA, Cohen AL, Power JD, Dosenbach NU, Church JA, Miezin FM, Schlaggar BL, Petersen SE (2009) Functional brain networks develop from a "local to distributed" organization. PLoS Comput Biol 5: e1000381. CrossRef Medline

Fair DA, Bathula D, Mills KL, Dias TG, Blythe MS, Zhang D, Snyder AZ, Raichle ME, Stevens AA, Nigg JT, Nagel BJ (2010) Maturing thalamocortical functional connectivity across development. Front Syst Neurosci 4:10. CrossRef Medline

Field AP (2001) Meta-analysis of correlation coefficients: a Monte Carlo comparison of fixed- and random-effects methods. Psychol Methods 6:161-180. CrossRef Medline

Fliesler SJ, Anderson RE (1983) Chemistry and metabolism of lipids in the vertebrate retina. J Lipid Res 22:79-131. Medline

Fox MD, Snyder AZ, Vincent JL, Corbetta M, Van Essen DC, Raichle ME (2005) The human brain is intrinsically organized into dynamic, anticorrelated functional networks. Proc Natl Acad Sci U S A 102:9673-9678. CrossRef Medline

Freeman MP, Hibbeln JR, Wisner KL, Davis JM, Mischoulon D, Peet M, Keck PE Jr, Marangell LB, Richardson AJ, Lake J, Stoll AL (2006) Omega-3 fatty acids: evidence basis for treatment and future research in psychiatry. J Clin Psychiatry 67:1954-1967. Medline

Guesnet P, Alessandri JM (2011) Docosahexaenoic acid (DHA) and the developing central nervous system (CNS): implications for dietary recommendations. Biochimie 93:7-12. CrossRef Medline

Hallquist MN, Hwang K, Luna B (2013) The nuisance of nuisance regression: spectral misspecification in a common approach to resting-state fMRI preprocessing reintroduces noise and obscures functional connectivity. Neuroimage 82:208-225. CrossRef Medline

He C, Qu X, Cui L, Wang J, Kang JX (2009) Improved spatial learning performance of fat- 1 mice is associated with enhanced neurogenesis and neuritogenesis by docosahexaenoic acid. Proc Natl Acad Sci U S A 106: 11370-11375. CrossRef Medline

Hoffman DR, Boettcher JA, Diersen-Schade DA (2009) Toward optimizing vision and cognition in term infants by dietary docosahexaenoic and arachidonic acid supplementation: a review of randomized controlled trials. Prostaglandins Leukot Essent Fatty Acids 81:151-158. CrossRef Medline

Hutchison RM, Everling S (2012) Monkey in the middle: why non-human primates are needed to bridge the gap in resting-state investigations. Front Neuroanat 6:29. CrossRef Medline

Hutchison RM, Leung LS, Mirsattari SM, Gati JS, Menon RS, Everling S
(2011) Resting-state networks in the macaque at 7 T. Neuroimage 56: 1546-1555. CrossRef Medline

Hutchison RM, Womelsdorf T, Gati JS, Leung LS, Menon RS, Everling S (2012) Resting-state connectivity identifies distinct functional networks in macaque cingulate cortex. Cereb Cortex 22:1294-1308. CrossRef Medline

Innis SM (2007) Dietary (n-3) fatty acids and brain development. J Nutr 137:855-859. Medline

Iyer SP, Shafran I, Grayson D, Gates K, Nigg JT, Fair DA (2013) Inferring functional connectivity in MRI using Bayesian network structure learning with a modified PC algorithm. Neuroimage 75:165-175. CrossRef Medline

Jeffrey BG, Neuringer M (2009) Age-related decline in rod phototransduction sensitivity in rhesus monkeys fed an n-3 fatty acid-deficient diet. Invest Ophthalmol Vis Sci 50:4360-4367. CrossRef Medline

Jeffrey BG, Mitchell DC, Gibson RA, Neuringer M (2002) n-3 fatty acid deficiency alters recovery of the rod photoresponse in rhesus monkeys. Invest Ophthalmol Vis Sci 43:2806-2814. Medline

Levant B, Zarcone TJ, Fowler SC (2010) Developmental effects of dietary n-3 fatty acids on activity and response to novelty. Physiol Behav 101: 176-183. CrossRef Medline

Lewis JW, Van Essen DC (2000) Corticocortical connections of visual, sensorimotor, and multimodal processing areas in the parietal lobe of the macaque monkey. J Comp Neurol 428:112-137. CrossRef Medline

Liang Z, King J, Zhang N (2012) Intrinsic organization of the anesthetized brain. J Neurosci 32:10183-10191. CrossRef Medline

Luchtman DW, Song C (2013) Cognitive enhancement by omega-3 fatty acids from childhood to old age: findings from animal and clinical studies. Neuropharm 64:550-565. CrossRef Medline

Mantini D, Gerits A, Nelissen K, Durand JB, Joly O, Simone L, Sawamura H, Wardak C, Orban GA, Buckner RL, Vanduffel W (2011) Default mode of brain function in monkeys. J Neurosci 31:12954-12962. CrossRef Medline

Margulies DS, Vincent JL, Kelly C, Lohmann G, Uddin LQ, Biswal BB, Villringer A, Castellanos FX, Milham MP, Petrides M (2009) Precuneus shares intrinsic functional architecture in humans and monkeys. Proc Natl Acad Sci U S A 106:20069-20074. CrossRef Medline

Martinez M (1992) Abnormal profiles of polyunsaturated fatty acids in the brain, liver, kidney and retina of patients with peroxisomal disorders. Brain Res 583:171-182. Medline

McLaren DG, Kosmatka KJ, Oakes TR, Kroenke CD, Kohama SG, Matochik JA, Ingram DK, Johnson SC (2009) A population-average MRI-based atlas collection of the rhesus macaque. Neuroimage 45:52-59. CrossRef Medline

McNamara RK, Able J, Jandacek R, Rider T, Tso P, Eliassen JC, Alfieri D, Weber W, Jarvis K, DelBello MP, Strakowski SM, Adler CM (2010) Docosahexaenoic acid supplementation increases prefrontal cortex activation during sustained attention in healthy boys: a placebo-controlled, dose-ranging, functional magnetic resonance imaging study. Am J Clin Nutr 91:1060-1067. CrossRef Medline

Meila M (2007) Comparing clusterings-an information based distance. J Multivar Anal 98:873-895. CrossRef

Mesulam MM, Mufson EJ (1982) Insula of the old world monkey. III. Efferent cortical output and comments on function. J Comp Neurol 212: 38-52. Medline

Moriguchi T, Greiner RS, Salem N Jr (2000) Behavioral deficits associated with dietary induction of decreased brain docosahexaenoic acid concentration. J Neurochem 75:2563-2573. CrossRef Medline

Mufson EJ, Mesulam MM (1982) Insula of the old world monkey. II: Afferent cortical input and comments on the claustrum. J Comp Neurol 212: 23-37. Medline

Neuringer M, Connor WE, Lin DS, Barstad L, Luck S (1986) Biochemical and functional effects of prenatal and postnatal omega 3 fatty acid deficiency on retina and brain in rhesus monkeys. Proc Natl Acad Sci U S A 83:4021-4025. Medline

Newman ME (2006) Modularity and community structure in networks. Proc Natl Acad Sci U S A 103:8577-8582. CrossRef Medline

O'Connor DL, Hall R, Adamkin D, Auestad N, Castillo M, Connor WE, Connor SL, Fitzgerald K, Groh-Wargo S, Hartmann EE, Jacobs J, Janowsky J, Lucas A, Margeson D, Mena P, Neuringer M, Nesin M, Singer L, Stephenson T, Szabo J, Zemon V, Zemon V (2001) Growth and development in preterm infants fed long-chain polyunsaturated fatty acids: 
a prospective, randomized controlled trial. Pediatrics 108:359-371. CrossRef Medline

O’Reilly JX, Croxson PL, Jbabdi S, Sallet J, Noonan MP, Mars RB, Browning PG, Wilson CR, Mitchell AS, Miller KL, Rushworth MF, Baxter MG (2013) Causal effect of disconnection lesions on interhemispheric functional connectivity in rhesus monkeys. Proc Natl Acad Sci U S A 110: 13982-13987. CrossRef Medline

Power JD, Cohen AL, Nelson SM, Wig GS, Barnes KA, Church JA, Vogel AC, Laumann TO, Miezin FM, Schlaggar BL, Petersen SE (2011) Functional network organization of the human brain. Neuron 72:665678. CrossRef Medline

Power JD, Barnes KA, Snyder AZ, Schlaggar BL, Petersen SE (2012) Spurious but systematic correlations in functional connectivity MRI networks arise from subject motion. Neuroimage 59:2142-2154. CrossRef Medline

Raz R, Gabis L (2009) Essential fatty acids and attention-deficithyperactivity disorder: a systematic review. Dev Med Child Neurol 51:580-592. CrossRef Medline

Reisbick S, Neuringer M, Hasnain R, Connor WE (1994) Home cage behavior of rhesus monkeys with long-term deficiency of omega-3 fatty acids. Physiol Behav 55:231-239. CrossRef Medline

Reisbick S, Neuringer M, Gohl E, Wald R Anderson, GJ (1997) Visual attention in infant monkeys: Effects of dietary fatty acids and age. Dev Psychol 33:387-395. Medline

Rubinov M, Sporns O (2011) Weight-conserving characterization of complex functional brain networks. Neuroimage 56:2068-2079. CrossRef Medline

Ryan AS, Astwood JD, Gautier S, Kuratko CN, Nelson EB, Salem N Jr (2010) Effects of long-chain polyunsaturated fatty acid supplementation on neurodevelopment in childhood: a review of human studies. Prostaglandins Leukot Essent Fatty Acids 82:305-314. CrossRef Medline

Saleem KS, Logothetis NK (2012) A combined MRI and histology atlas of the Rhesus monkey brain in stereotaxic coordinates, 2nd edition. San Diego: Academic.

Salvador R, Suckling J, Coleman MR, Pickard JD, Menon D, Bullmore E (2005) Neurophysiological architecture of functional magnetic resonance images of human brain. Cereb Cortex 15:1332-1342. CrossRef Medline
SanGiovanni JP, Berkey CS, Dwyer JT, Colditz GA (2000) Dietary essential fatty acids, long-chain polyunsaturated fatty acids, and visual resolution acuity in healthy fullterm infants: a systematic review. Early Hum Dev 57:165-188. CrossRef Medline

Savazzi S, Fabri M, Rubboli G, Paggi A, Tassinari CA, Marzi CA (2007) Interhemispheric transfer following callosotomy in humans: role of the superior colliculus. Neuropsychologia 45:2417-2427. CrossRef Medline

Simopoulos AP (2011) Importance of the omega-6/omega-3 balance in health and disease: evolutionary aspects of diet. World Rev Nutr Diet 102:10-21. CrossRef Medline

Stevens AA, Tappon SC, Garg A, Fair DA (2012) Functional brain network modularity captures inter- and intra-individual variation in working memory capacity. PLoS One 7:e30468. CrossRef Medline

Tixier-Vidal A, Picart R, Loudes C, Bauman AF (1986) Effects of polyunsaturated fatty acids and hormones on synaptogenesis in serum-free medium cultures of mouse fetal hypothalamic cells. Neuroscience 17: 115-132. Medline

Uddin LQ, Mooshagian E, Zaidel E, Scheres A, Margulies DS, Kelly AM, Shehzad Z, Adelstein JS, Castellanos FX, Biswal BB, Milham MP (2008) Residual functional connectivity in the split-brain revealed with restingstate functional MRI. Neuroreport 19:703-709. CrossRef Medline

Vincent JL, Patel GH, Fox MD, Snyder AZ, Baker JT, Van Essen DC, Zempel JM, Snyder LH, Corbetta M, Raichle ME (2007) Intrinsic functional architecture in the anaesthetized monkey brain. Nature 447:83-86. CrossRef Medline

Wainwright PE (2002) Dietary essential fatty acids and brain function: a developmental perspective on mechanisms. Proc Nutr Soc 61:61-69. CrossRef Medline

Yeo BT, Krienen FM, Sepulcre J, Sabuncu MR, Lashkari D, Hollinshead M, Roffman JL, Smoller JW, Zöllei L, Polimeni JR, Fischl B, Liu H, Buckner RL (2011) The organization of the human cerebral cortex estimated by intrinsic functional connectivity. J Neurophysiol 106:1125-1165. CrossRef Medline

Zhang D, Snyder AZ, Shimony JS, Fox MD, Raichle ME (2010) Noninvasive functional and structural connectivity mapping of the human thalamocortical system. Cereb Cortex 20:1187-1194. CrossRef Medline 